\title{
Beat Gerber
}

\section{Im Spannungsfeld zwischen Tun und Lassen - ein medizinphilosophischer Beitrag}

In einem ersten Teil dieser Arbeit werden drei Begriffe erörtert, die in der Medizin immer wieder zu unterschiedlichen Interpretationen und Diskussionen führen und die wichtig für das Verständnis meiner weiteren Überlegungen sind. Es geht um die Begriffe Gesundheit und Krankheit (1.1), Medikalisierung (1.2), und Macht in der Medizin (1.3). In einem zweiten Teil soll die Thematik des Tuns und Lassens in der Medizin behandelt werden. Ich beginne mit zwei handlungstheoretischen Optionen (2.1) und diskutiere das Handeln oder Tun in der Medizin (2.2) sowie das (situative) Unterlassen medizinischer Interventionen (2.3), dessen Risiken und Chancen anschliessend untersucht werden. Ganz besonders interessiert die Frage, was wir tun wir, wenn wir medizinisch nichts tun (2.5). ${ }^{1}$

Zwei Vorbemerkungen: 1) Das Verständnis von Krankheit und Medizin, das dieser Arbeit zugrunde liegt, ist das eines praktizierenden Arztes, der sich nach fast vierzig Jahren klinischer Tätigkeit mit seinem Beruf kritisch auseinandergesetzt und die ärztliche Praxis philosophisch reflektiert hat. Es handelt sich also um eine Art philosophische Supervision der Medizin. Die philosophische Sicht auf Gesundheit, Krankheit und Medizin führt einerseits rasch zu kritischen Fragen, zu neuen Überlegungen und sehr oft zu kontroversen Diskussionen. Andererseits geht es hier aber auch um eine kritische

1 Für eine ausführliche Behandlung dieser Thematik vgl. Beat Gerber: Warum die Medizin die Philosophie braucht. Für ein umfassendes Verständnis von Krankheit und Medizin, Bern 2020. 
Hinterfragung der eigenen beruflichen Tätigkeit und insbesondere um die Thematik des Tuns und Unterlassens medizinischer Handlungen. Um es gleich vorwegzunehmen: Ich bin der Meinung, dass gewisses Tun in der Medizin besser zu unterlassen wäre. Ich versuche im Folgenden aufzuzeigen, weshalb die Option der situativen Unterlassung von diagnostischen und therapeutischen Interventionen zwingend $\mathrm{zu}$ jeder patientenorientierten, genuinen Medizin gehört. In der modernen Medizin ist sehr oft das Tun das übliche, das Nicht(s)tun dagegen die Ausnahme. Ich werde aufzeigen, weshalb diese Praxis heute die Regel ist, dass sie in vielen Fällen jedoch für den Patienten nicht zwingend die beste Lösung ist. Dabei geht es explizit nicht um ökonomische Überlegungen oder Sparmassnahmen. Diese sind zwar absolut notwendig, aber sie sind hier nicht das Thema.

2) Wenn hier von «Medizin» die Rede ist, geht es immer um die klassische, westlich geprägte Schulmedizin. Das zu wissen ist für das Verständnis der weiteren Ausführungen wichtig.

\section{Drei zentrale Begriffe}

\subsection{Gesundheit und Krankheit}

Was genau man unter Gesundheit und Krankheit versteht, ist oft unklar. Der Inhalt dieser Begriffe wird stark durch Sprache, Kultur und Geschichte geprägt und ganz unterschiedlich interpretiert. Zudem implizieren die beiden Begriffe biologisch-naturwissenschaftliche und anthropologisch-geisteswissenschaftliche Aspekte, die wiederum individuell und gesellschaftlich unterschiedlich wahrgenommen und gewertet werden. Dies zeigt sich beispielsweise daran, dass verschiedenste Disziplinen (Medizin, Jurisprudenz, Philosophie, Theologie, Versicherungen) sich schwertun, wenn es darum geht, sich auf eindeutige Definitionen zu einigen. Und auch innerhalb dieser Disziplinen existieren - abhängig von kulturellen und gesellschaftspolitischen Vorgaben - oft divergierende Vorstellungen von Gesundheit und Krankheit. 
Hinzu kommt, dass sich die Begriffe «gesund» und «krank» zunehmend destabilisieren. Der Übergang von gesund zu krank wird zu einer immer breiteren Grauzone, die Grenze dazwischen wird unscharf und löst sich auf. Diese Grenzziehung ist ein dynamischer Prozess, die Demarkationslinie verschiebt sich ständig: Wer früher noch gesund war, ist heute bereits krank. Das Spektrum des Anormalen und Pathologischen wächst in einem Ausmass, wie das Spektrum des Normalen und Gesunden schrumpft. Beides ist Ausdruck einer zunehmenden Medikalisierung. Zudem ist vieles, was in irgendeiner Weise vom Normalen abweicht, nicht nur pathologisch, es wird oft auch korrigiert, also behandelt. Eine solche grundsätzliche Skepsis gegenüber all dem, was sich in einer Grauzone befindet, ist jedoch heikel, denn sie kann zur Ausgrenzung des Andersartigen, zur Stigmatisierung des nicht ganz Normalen und zur Intoleranz gegenüber allem führen, was nicht üblich ist.

Es gibt viele Definitionen von Gesundheit und Krankheit. Was Nietzsche beispielweise als die "grosse Gesundheit» bezeichnete, ${ }^{2}$ ist ein Bereich, in welchem eine Person fähig ist, «in vollen Zügen zu leben, so dass sie immer wieder an ihre existentiellen Grenzen gelangt.» ${ }^{3}$ Das Alltägliche wird dabei überschritten, und es kann sich das Gefühl von Sinnhaftigkeit einstellen. Die grosse Krankheit dagegen erleben die Betroffenen als existentielle Krise, eine Krise, «die sie an ihre Abhängigkeit vom guten Willen, der Anteilnahme und Zuneigung anderer Menschen mahnt und sie in Kontakt mit dem Tod und mit der spirituellen Dimension des menschlichen Lebens bringt.» ${ }^{4}$ Es kommt dabei zur Grenzerfahrung, die ebenfalls das Gefühl von Sinnhaftigkeit der eigenen Existenz wachrufen kann. Die kleine Gesundheit ist gleichbedeutend mit der Abwesenheit von Krankheit und beinhaltet auch Zustände erhöhter Krankheitsgefährdung und das Gefühl von anhaltender Sinnlosigkeit. Mit kleiner gabe, hg. von Giorgio Colli und Mazzino Montinari, V 635-637.

3 Piet van Spijk: Krankheit, Gesundheit, Religion und Spiritualität, in: Schweizerische Ärztezeitung 94/ 6 (2013) 224-225.

Ebd. 
Krankheit dagegen sind die vielen gesundheitlichen Probleme, die sogenannten banalen Krankheiten gemeint. ${ }^{5}$

Für eine umfassende Definition von Gesundheit und Krankheit sind meiner Ansicht nach zwei Aspekte zentral: Erstens geht es explizit um die Krankheit des Menschen - mit all seinen kulturellen Werten. Und es geht um den Menschen als biologisches Wesen, das immer ein denkendes und fühlendes Wesen ist. Diese Definition steht demnach auf einer anthropologischen Grundlage. Und zweitens geht es bei dieser Definition um das Faktum der Kontingenz, um den Zufall. Oft handelt es sich dabei um den vordergründigen und vermeintlichen Zufall, gesund zu sein - respektive von einer Krankheit verschont oder eben nicht verschont zu bleiben. Gesundheit und Krankheit sind demnach dem Menschen kontingent. Wer gesund ist, der hat Glück gehabt, und wer krank ist, nicht. Und was bleibt, ist immer auch ein gewisses Mass an Unverfügbarkeit. ${ }^{6}$

Gesunde und insbesondere kranke Menschen sind somit immer eingebettet in einen nur sie betreffenden Zusammenhang: Es ist ihre Art gesund und krank zu sein, es ist ihre persönliche Geschichte, ihr soziales Umfeld und auch ihre Zukunft. Es geht um das Begreifen und Verstehen des Kranken in naturwissenschaftlicher und kultureller Hinsicht. Und es geht dabei auch um den Aspekt der Kontingenz, um integrale Grunderfahrungen, die jeder Mensch im Laufe seines Lebens ungewollt und ungefragt macht. Man könnte auch von Ordnungsbrüchen sprechen, die jeder Einzelne als Unverfügbarkeit erlebt - in ihrer negativen Form als Krankheiten, persönliche Leiden.

Gesundheit und Krankheit werden somit konstituiert durch physische, psychische, gesellschaftliche und andere Faktoren und sie sind als Zusammenspiel und Ergebnis überaus komplexer interner und externer Prozesse zu verstehen. Diese Prozesse sind zwar im Wesentlichen kausal, aber nicht immer. Und falls Kausalität herrscht, ist eine solche in sehr vielen Fällen weder dem Betroffenen noch dem Beobachter wirklich bekannt. Ein grosser Teil dieses

$5 \quad$ Vgl. ebd.

6 Vgl. B. Gerber: Warum die Medizin die Philosophie braucht, 51-52. 
prozessualen Geschehens bleibt deshalb mindestens vordergründig kontingent.

Der Praxisalltag zeigt nun aber, dass die in der modernen Medizin anerkannten Begrifflichkeiten von Gesundheit und Krankheit überwiegend biologisch-naturwissenschaftlicher Art sind, von einer durchgehenden Kausalität und prinzipiellen Berechenbarkeit ausgehen und den Aspekt der erlebten Kontingenz weitgehend verdrängen. Ebenso fällt es ihr oft schwer, anthropologische und kulturelle Werte in ihr Begriffsverständnis einzubinden. Einer Medizin aber, die diese Einseitigkeit ihres Denkens weiterhin zulässt, wird es nur schwer gelingen, dem Kranken als Menschen zu begegnen und ihm in all seinen Dimensionen gerecht zu werden.

Dass trotz weitgehender Unverfügbarkeit im Zusammenspiel von Gesundheitserhaltung und Krankheitsentwicklung immer auch ein wesentlicher Anteil an Selbstbestimmbarem und Selbstverursachtem mit dabei ist, ist evident. Die Bedeutung der Mitverantwortung für die eigene Gesundheit ist demnach nicht zu unterschätzen. Die Vorstellung jedoch, die von gewissen Gesundheitspolitikern und Versicherern zunehmend vertreten wird, dass das Individuum für seine Krankheit letztlich allein und selbst verantwortlich sei, ist sachlich falsch und grundsätzlich abzulehnen.

Damit sehen wir uns mit dem Aspekt der Solidarität in der Medizin konfrontiert. Sie kommt am ehesten bei unverschuldetem Leid auf - sei dies nun im Kontext einer Unwetterkatastrophe oder eben infolge eines unerwarteten Krankheitsausbruchs. Dennoch lässt sich eine solche für eine aufgeklärte Gesellschaft unabdingbare Solidarität oft vermissen. Für Samia Hurst, Bioethikerin in Genf, besteht die Tendenz, dass heute oft der Mensch selbst verantwortlich gemacht und dass Krankheit als persönliches Versagen angesehen werde: «Die Raucherin ist 〈schuld〉 an ihrem Lungenkrebs, oder der Fettleibige an seinem Diabetes und Eltern haben Schuld am Erb-Leiden ihres Kindes, dessen Geburt sie nicht verhindert haben. Nun sollen die betroffenen dafür bezahlen.» Das ist falsch und unethisch. «Die Medizin ist keine moralische Instanz, ihre Logik ist eine andere als die der Strafe. Es ist sehr gefährlich, 
Gesundheitspolitik mit den Prinzipien der Strafe zu verknüpfen. Das müssen wir verhindern.» ${ }^{7}$

\subsection{Medikalisierung}

Es ist der Mensch selbst, der im Laufe der Zeit die Begrifflichkeiten von Gesundheit und Krankheit ständig hinterfragt hat und neu zu definieren suchte. Und er ist es auch, der zum unaufhaltsamen Prozess der Medikalisierung und zur Ausweitung des Einflussbereichs der Medizin beigetragen hat. Der Mensch tut dies unter anderem, weil die Einlösung des Versprechens der modernen Medizin auf mehr Gesundheit und weniger Krankheit eine immer exaktere Diagnostik erfordert und bessere Therapiemöglichkeiten erlaubt. Und er tut dies auch mit der hehren Absicht, dem Krankgeschriebenen Schutz und Unterstützung zukommen zu lassen.

Unter Medikalisierung wird ein übermässiges Ausbreiten der Medizin in Einflussbereiche verstanden, die ursprünglich mit Medizin wenig oder gar nichts zu tun haben. Sie sieht sich heute in vielen gesundheitlichen, juristischen und gesellschaftlichen Gebieten zuständig, in die sie früher nicht involviert war: Dazu gehören beispielsweise gewisse Formen von Befindlichkeitsstörungen und Verhaltensauffälligkeiten, Bereiche wie gesundheitliche Vorbeugung und Gesundheits-Screening, Fitness und Ernährung, aber auch die meisten Suchtkrankheiten. Sie alle standen früher nicht unter dem Einflussbereich der Medizin.

Der Begriff der Medikalisierung war primär ein rein deskriptiver und somit grundsätzlich wertneutral. Heute hat er im Zuge der kritischen Ausdehnung und Einflussnahme diese Wertneutralität weitgehend verloren. Medikalisierung geschieht mehrheitlich im Konsens mit Individuum und Gesellschaft: Sie verschafft all jenen Schutz, die sich irgendwo im Grenzbereich zwischen gesund und krank verorten. Ihr gesundheitlicher Zustand wird neu als krank definiert, was Anspruch auf Hilfe verspricht und entsprechend 1584. 
medizinische, soziale und finanzielle Unterstützung legitimiert. Solches gehört selbstverständlich zu den Errungenschaften eines modernen Staates und ist grundsätzlich begrüssenswert.

Trotzdem ist eine Medikalisierung in diesem Ausmass, insbesondere die offenkundige Ausweitung des Krankheitsbegriffs, nicht unproblematisch. Gerade weil die Grenzen zwischen gesund und krank unscharf sind und Raum zur Interpretation bieten, muss jemand die Definitionsmacht übernehmen. Doch wer ist dazu legitimiert? Die Medizin ist hier Partei und somit nicht unvoreingenommen. Diese Parteilichkeit führt erwartungsgemäss eher zu einer Expansion des Zuständigkeits- und Einflussbereichs der Medizin als zu deren Reduktion, und noch viel weniger zu einer Selbstzensur oder Selbstbegrenzung. Insbesondere auf der Seite der Anbieter tut sich im Zuge fortschreitender Medikalisierung ein breites Feld auf sie alle sind gewillt, die sich dabei öffnenden Marktlücken rasch zu schliessen. Und sie tun dies in einem Ausmass, das die Vermutung zulässt, dass sich das Ganze durchaus auch rechnet - jedenfalls für sie selbst.

Der Zürcher Theologie und Ethiker Johannes Fischer hat sich kritisch mit solchen Fragen auseinandergesetzt und ausführlich dazu geäussert. Dabei stellte er mehrere Fragen: Welche gesundheitliche Beeinträchtigung wird schon und welche noch nicht als Krankheit angeschaut? Wie können überzogene und ausufernde Vorstellungen von «sozialer Gerechtigkeit» zurückgewiesen werden ${ }^{8}$ Ein massloses Anspruchsdenken ist eine der wichtigsten Triebfedern für die Medikalisierung. Kann und soll der Medikalisierung durch die Ärzte entgegengewirkt werden? Die Ursache liegt hier oft in der direkten Konfrontation des Arztes mit der Erlebnisperspektive des Patienten und dem daraus resultierenden Bedürfnis des Arztes, dem Patienten zu helfen. Können und sollen die Patienten selbst der Medikalisierung entgegenwirken? Die Einstufung der meisten gesundheitlichen Störungen als Krankheit ist unbestritten und für die Betroffenen in den meisten Fällen entlastend.

8 Johannes Fischer: Ethische Aspekte der Medikalisierung, in: Bioethica Forum 5/2 (2012) 69-72. 
Es ist aber auch bekannt ist, dass das Delegieren solcher Beeinträchtigungen an die Medizin gelegentlich der Selbstverantwortung und der Selbstsorge des Patienten nachhaltig abträglich ist.

Das unaufhaltsame Vordringen der Medizin in die persönlichen und sozialen Bereiche des Lebens hat dazu geführt, dass diese noch nie so mächtig war, wie sie es heute ist. Und damit kommen wir zu einem Paradoxon der modernen Medizin: Statt sich nun sicherer und gesünder zu fühlen, werden viele dieser faktisch gesunden Menschen krank, weil sie sich um ihre Gesundheit sorgen. Der Widerspruch liegt darin, dass, je wohlhabender ein Land ist und je mehr finanzielle Mittel dessen Bevölkerung für ihr Gesundheitssystem bereitstellt, desto kranker fühlt sich diese. Die Verunsicherung und die damit verbundenen Sorgen um die eigene Gesundheit nehmen in bedenklicher Weise zu. Gemäss Untersuchungen von Amartya Sen sollen Bildungsniveau und Selbstbeobachtung in direktem Zusammenhang stehen: Je höher das Bildungsniveau ist, desto mehr beschäftigen sich die Menschen mit ihren Unpässlichkeiten, Beschwerden und Gebresten. ${ }^{9}$

So sind wir wieder bei der anthropologischen Grundfrage: Was ist der Mensch? Was ist Gesundheit und Krankheit. Und was ist und soll die Medizin denn eigentlich? Und so geht es letztlich wieder um die Frage nach dem Selbstverständnis des Menschen und um sein Verständnis von Gesundheit, Krankheit und Medizin.

\subsection{Macht in der Medizin}

Die Thematisierung der Macht im Kontext von Tun und Lassen in der Medizin ist sinnvoll und geschieht aus drei Gründen. Erstens aus praktischen Gründen, denn wir müssen wissen, wer die Macht besitzt. Es sind dies die Ärzteschaft und die Pflege, das Krankenhaus, die Krankenversicherung, die Gesellschaft, aber auch der Patient selbst. Zweitens aus moralischen Gründen, denn wer die (2002) 860-861. 
Macht besitzt, muss die Verantwortung für seine Entscheidungen übernehmen. Und drittens aus Gründen der Beurteilung und Evaluation, denn wir müssen wissen, ob Macht für irgendeine bestimmte missliche Situation ursächlich ist - und damit eine Mitschuld trägt.

Nach Max Weber ist Macht die Chance, «innerhalb einer sozialen Beziehung den eigenen Willen auch gegen Widerstreben durchzusetzen, gleichviel, worauf diese Chance beruht $\gg .{ }^{10}$ Damit gemeint ist Macht als Willensdurchsetzung. Hier stellt sich immer und zwingend die Legitimationsfrage. Im Gegensatz dazu versteht Hannah Arendt Macht als «Handeln mit anderen in gegenseitigem Einvernehmen»: «Macht entspricht der menschlichen Fähigkeit, nicht nur zu handeln oder etwas zu tun, sondern sich mit andern zusammenzuschliessen und im Einvernehmen mit ihnen zu handeln.» ${ }^{11}$ Für Hannah Arendt ist Max Webers Macht als Herrschaft geradezu das Gegenteil von Macht.

Im medizinischen Alltag sind beide Arten von Macht präsent. Die erste Form nach Weber ist patientenfeindlich und im Kontext von Krankheit und Medizin meistens kontraproduktiv, die zweite nach Arendt ist dagegen patientenfreundlich und produktiv. Unser aktuelles Medizinverständnis und unser mehrheitlich als partnerschaftlich verstandenes Arzt-Patienten-Verhältnis zwingt die Akteure in der Medizin dazu, Macht als Handeln «im Einvernehmen» $\mathrm{zu}$ verstehen und entsprechend auszuüben. Dennoch wird Macht nicht selten als Willensdurchsetzung verstanden, insbesondere in patriarchalisch geprägten Arzt-Patienten-Verhältnissen.

Macht ist in der Medizin omnipräsent und äussert sich verschiedenartig. Martin Hartmann beispielsweise erwähnt (1) die Definitionsmacht (über gesund und krank, über den Dringlichkeitsgrad respektive über die Aufnahme oder Nichtaufnahme ins Krankenhaus, aber auch die Macht zur Bagatellisierung eines Leidens bei Fehlen von naturwissenschaftlich belegbaren Fakten); (2) die Sanktionsmacht (beispielsweise Atteste und Gutachten); (3) die

10 Max Weber: Wirtschaft und Gesellschaft, Tübingen 2002, Kapitel 1, § 16.

11 Hannah Arendt: Macht und Gewalt, München 1993, II 44-58. 
Steuerungsmacht (beispielsweise die Kompetenz zur Steuerung der Gesprächsführung, der Abklärungs- und Behandlungsstrategie); und (4) die institutionelle Macht (Statusakzeptanz, Anonymität, Warteschlangen, Patienten als Bittsteller oder die Position gegenüber Krankenversicherungen und Institutionen). ${ }^{12}$

Krankheit führt unweigerlich zu einem Ungleichgewicht zwischen dem Heilenden und dem zu Heilenden und geht oft zu Lasten des zu Heilenden. Es kommt aber auch vor, dass die Machtverhältnisse umgekehrt sind: Ein übermüdeter und überforderter Arzt steht in einer Notfallsituation den Begehrlichkeiten und Erwartungen eines anspruchsvollen Patienten gegenüber. Hier sind einfach die Rollen vertauscht. Dies geschieht im Übrigen viel häufiger, als von Patientenseite vermutet wird.

Wenn Macht in der Medizin im Sinne von Hannah Arendt zu verstehen ist, nämlich als ein erwünschtes Ergebnis einer nützlichen und erfolgreichen Zusammenarbeit zwischen dem Patienten und dem Arzt, dann ist Macht weit davon entfernt, als Bedrohung oder Gefahr aufzutreten. In dieser Form ist sie geeignet, für den Patienten die richtigen Entscheide zu treffen.

\section{Tun und Lassen in der Medizin}

Um das Spannungsfeld von Tun und Lassen in der Medizin sichtbar zu machen, ist die Kenntnis wesentlicher Fakten zu der breiten Thematik von Gesundheit, Krankheit und Medizin zwingend. Zentral sind hier die drei im ersten Teil vorgestellten und diskutierten Themenkreise: Was ist Gesundheit, was Krankheit? Was bedeutet Medikalisierung? Und wo und in welcher Art äussert sich Macht in der Medizin?

In diesem zweiten Teil geht es nun um das Tun, um das Handeln in der Medizin und insbesondere um die Option des situativen 
Nicht(s)tuns. In der modernen Medizin ist bekanntlich das Tun das übliche, denn meistens ist ja auch etwas zu tun. Das Nicht(s)tun, das absichtliche Unterlassen einer bestimmten medizinischen diagnostischen oder therapeutischen Intervention dagegen ist erfahrungsgemäss die Ausnahme. Um genau dieses situative Nicht(s)tun in der Medizin geht es im Folgenden. Man kann hier einwenden, dass heute aufgrund des hohen Kostendrucks in vielen Fällen auch zu wenig oder überhaupt nichts mehr getan wird. Das mag für einzelne Fälle zutreffen - dann handelt es sich aber nicht um einen bewussten, freiwilligen Entscheid zur Unterlassung einer bestimmten medizinischen Intervention aufgrund der Überzeugung, dass das Nicht(s)tun für den Patienten die bessere Lösung ist, sondern bloss um einen Handlungsverzicht zwecks Kostenersparnis.

Bei der Thematik des situativen Nicht(s)tuns in der Medizin, von dem hier die Rede ist und das explizit ein situatives ist, geht es um die Möglichkeit der freien Entscheidung in einer Wahl zwischen den beiden zur Verfügung stehenden Optionen des Handelns und des Nicht-Handelns. Und gewählt wird dabei der Handlungsverzicht, weil sowohl der Patient als auch der Arzt der Überzeugung sind, dass nur ein solcher dem Patientenwohl wirklich dient. Es ist der Aspekt der situativen Unterlassung, diese kleine Nische, die sonst niemanden interessiert, eine Unterlassung, die im Übrigen im Praxisalltag gar nicht so einfach ist, denn das Handeln steht uns wesentlich näher als das Nicht-Handeln. Nebst hoher Wertschätzung und grossem Respekt vor all den Spitzenleistungen der modernen Medizin macht sich bei Gesunden und Kranken ein gewisses Unbehagen bemerkbar. Es wird generiert durch die Beobachtung, dass das enorme Potential an medizinisch-technischen Möglichkeiten dem genuin Menschlichen in der Medizin oft abträglich ist. Was wir brauchen, ist eine ausgewogene Praxis des Handelns und des Geschehen-lassens, eine Praxis, die für den Patienten das Optimale, nicht das Maximale im Visier hat.

Und es geht in diesem zweiten Teil auch darum, aufzuzeigen, dass die im ersten Teil vorgestellten Themenkreise eine zentrale Rolle spielen: nämlich immer dann, wenn es darum geht, im praktischen 
Alltag zu entscheiden, ob aus medizinischer Sicht etwas zu tun oder eben zu unterlassen ist.

\subsection{Handlungstheoretische Optionen}

Handlungstheoretisch gibt es zwei Optionen: Handeln und nicht handeln. Was hier interessiert, sind im Wesentlichen zwei Fragen in Zusammenhang mit dem Nicht-handeln, also mit der Unterlassung einer Handlung.

Erstens die Frage der kausalen Wirksamkeit des Unterlassens einer Handlung: Kann etwas von seiner Wesens- und Begriffsbestimmung her Negatives denn irgendetwas verursachen oder gar bewirken? Die Antwort lautet ja. Ontologisch Negatives negiert den Aspekt der Kausalität nicht, denn die Unterlassung einer Handlung unterwirft sich der Kausalität des Spontanverlaufs.

Zweitens zur Frage der moralischen Bedeutsamkeit des Unterlassens einer Handlung: Kann man sich durch Unterlassung einer Handlung aus der Verantwortung stehlen? Nein, denn sowohl für das Handeln als auch für das Unterlassen, also für das Zulassen des Spontanverlaufs, braucht es gute Gründe. Die Antwort lautet: Bezüglich moralischer Bedeutsamkeit gibt es keinen prinzipiellen Unterschied zwischen Handeln und Unterlassen. Das heisst, dass sich der Unterlassende, der es einfach geschehen lässt, sich weder der Kausalität noch der moralischen Bedeutsamkeit und Verantwortlichkeit entziehen kann.

\subsection{Das Handeln oder Tun in der Medizin}

Was hier interessiert, ist das Tun und Nicht(s)tun im Kontext der Medizin. Das Kerngeschäft ärztlichen Handelns wird heute getrieben durch permanente Interventionen am Kranken. Dass medizinischer Aktivismus in vielen Fällen notwendig ist und dass sich die ärztliche Praxis im Wesentlichen über das Tun definiert, steht hier nicht zur Debatte. Es geht vielmehr um die Feststellung, dass in der Medizin oft ein medizinischer Hyper-Aktivismus in Form permanenter medizintechnischer Interventionen betrieben wird, und dass dieser 
in vielen Fällen nicht zum Wohl des Patienten ist. Aktivismus in der Medizin wird im Wesentlichen durch drei Faktoren gefördert:

(1) Durch die treibenden Kräfte: Auf Seiten des Patienten sind es die allzu grossen Erwartungen an die Medizin und auf Seiten des Arztes sein Pflichtgefühl und seine Angst vor Misserfolg und vor allfälligen juristischen Konsequenzen. Dazu kommen überhöhte Machbarkeitsvorstellungen, Selbstüberschätzung, finanzielle Fehlanreize und Interessen der Wissenschaft.

(2) Durch eingespielte Automatismen: Dazu gehören diagnostische und therapeutische Aktionen, die sich in einer bestimmten Situation gewohnheitsmässig, gedankenlos, algorithmisch und zwangsläufig vollziehen. Sie werden im Einzelfall kaum hinterfragt. So beispielsweise Routineuntersuchungen wie gewisse Labortests, Röntgen- und Ultraschalluntersuchungen, aber auch andere medizinisch-technische Abklärungen. Automatismen sind in der Medizin häufig und nicht generell schlecht. Sie sind Ausdruck einer gewissen beruflichen Routine und Erfahrung, können aber auch Folge eines ständigen Zeitdrucks und fachlicher Unsicherheit sein. Vor allem aber verhindern Automatismen die Möglichkeit, in einer bestimmten Situation etwas anderes oder gar nichts zu tun.

(3) Durch fragwürdige Abhängigkeiten: Übermässiges Tun kann auch durch problematische Abhängigkeiten gefördert werden, insbesondere im Spitalbereich. Sie entstehen in erster Linie im Dreiecksverhältnis von Leistungsempfänger, Leistungserbringer und Leistungsträger. Und bekanntlich ist auch das Verhältnis zwischen den Ärzten verschiedener Hierarchiestufen nicht immer ganz unproblematisch.

Das Zusammenspiel dieser treibenden Kräfte, Automatismen und Abhängigkeiten in Verbindung mit dem Faktor Macht als Willensdurchsetzung trägt entscheidend zum auffallend aktiven Verhalten der Ärzte und Patienten bei. Für sie alle gilt der Handlungsprimat, das heisst, sie führen zu permanentem medizinischtechnischen Handeln. Und sie alle haben unmittelbar mit den drei inzwischen bekannten Fragen zu tun, was mit Gesundheit und Krankheit überhaupt gemeint ist, wieso sich oft eine Medikalisierung 
im Übermass ergeben kann und wieso letztlich auch Machtfaktoren in der Medizin zum Wirken kommen.

2.3 Das situative Unterlassen medizinischer Interventionen.

Situatives Nicht(s)tun in der Medizin

Der Ausdruck «situatives Nicht(s)tun in der Medizin» meint die Unterlassung medizinischer Vereinnahmungen des Patienten durch Diagnostik, Therapie und andere Interventionen, die dem Wohlergehen des Patienten abträglich sind. Insbesondere geht es darum, dem Patienten physische und psychische Kollateralschäden von nutzlosen Interventionen zu ersparen. Die vordringliche Aufgabe der Medizin ist es, das Sinnvolle und Nützliche für den Kranken zu erkennen und sich vom Sinnlosen und Nicht-Nützlichen konsequent zu verabschieden. Das Adjektiv «situativ» weist darauf hin, dass im Laufe des ganzen Abklärungs- und Behandlungsprozesses genau hinzuschauen ist und diejenigen Situationen zu benennen sind, in denen die Unterlassung einer medizinisch-technischen Intervention für den Patienten die bessere Option ist. An diesem Findungsprozess sind immer beide, der Arzt und der Patient, beteiligt.

Das Unterlassen medizinischer Interventionen ist explizit immer dann geboten, wenn Interventionen jeglicher Art für das leibliche oder seelische Wohl des Patienten kontraproduktiv sind. Oder anders formuliert: Schlecht begründete, unnötige Diagnostik und Therapie, die den Patienten nur belasten und ihm keinen Nutzen bringen, sind zwingend zu unterlassen. Die Forderung nach situativem Nicht(s)tun lässt sich begründen. Einerseits rational: Wenn davon auszugehen ist, dass der Spontanverlauf für den Patienten die bessere Alternative ist, besteht die Pflicht, medizinisches Tun zu unterlassen. Situatives Nicht(s)tun fordert dementsprechend einerseits einen normativen Status im Sinn einer ärztlichen Verpflichtung. Andererseits lässt sich diese Forderung auch durch die Autonomierechte der Patienten begründen: namentlich durch das Gebot, den Patienten vor nicht zwingenden oder gar unnötigen medizinisch-technischen Vereinnahmungen fernzuhalten und $\mathrm{zu}$ schützen. Es soll dem 
Patienten unersetzbare Freiräume verschaffen, ihm medizinexterne Handlungsweisen ermöglichen und ihn in Frieden und Ruhe leben zu lassen, was es noch zu leben gibt.

Für die Konzeption des situativen Nicht(s)tuns in der Medizin sind die Aspekte der Intuition und der Intention fundamental. Intuition hat viel mit Ahnung und Gespür zu tun, beides Qualitäten, die dem Subjektiven sehr nahestehen. Intuition ergibt sich aus hinschauen, betrachten und erwägen und steht für ein spontanes, umfassendes Wahrnehmen einer bestimmten Gegebenheit. Intuition ist zwar dem Risiko der Täuschung unterworfen, bildet jedoch die individuellen und unmittelbaren Bedürfnisse des Betroffenen ab. Intention meint die Absicht und die Bereitschaft, etwas zu tun oder zu lassen. Diese Absichtlichkeit impliziert die Bereitschaft, für eine bestimmte Entscheidung selbst die Verantwortung zu übernehmen und ist darüber hinaus ein wesentliches Element der Patientenautonomie.

2.4 Risiken und Chancen von Tun und Nicht(s)tun in der Medizin

Beide, das Tun und das Unterlassen sind risikoreich, beide implizieren Chance und Gefahr zugleich. Ihnen gemeinsam ist zudem ein Paradoxon - nämlich das Paradoxon, dass beide Optionen vorgeben, in einem bestimmten Fall die bessere Position zu sein, ihre antagonistische Alternative aber in der Praxis nie gleichzeitig realisiert werden kann. Das Ergebnis der Alternative wird somit nie bekannt sein.

Jedes Tun, jedes medizinische Handeln ist riskant und janusköpfig, denn auch wenn die Gründe für das Tun gute Gründe sind, entspricht das tatsächliche Handlungsergebnis nicht in jedem Fall dem erhofften - das zeigt die Alltagserfahrung. Wenn nun - trotz guten Handlungsgründen - das Ergebnis «schlecht» ist, wird man zwar enttäuscht sein, man wird sich aber damit trösten, zumindest etwas gemacht und nichts versäumt zu haben. Gleich einem Handlungsversuch, der nun eben gescheitert ist.

Im Fall der Unterlassung einer medizinischen Handlung dagegen ist dies ganz anders, denn es kommt rasch einmal der Gedanke 
auf, nun doch etwas verpasst zu haben. Genau hier liegt eine der Schwierigkeiten des situativen Nicht(s)tuns in der Medizin, und sie kann zum Stolperstein für deren Akzeptanz werden. Bei Handlungsunterlassung stellt sich demnach ganz besonders und schon sehr früh die Frage, wie ein allenfalls schlechter Krankheitsverlauf vom Patienten und dessen Angehörigen wohl akzeptiert wird. Hier besteht das Risiko, dass im Fall eines ungünstigen Verlaufs eine Unterlassung im Nachhinein kritisiert wird. Auch dann, wenn gar nie bekannt sein wird, ob sich das Tun wirklich als geeigneter erwiesen hätte. Aus diesem Grund ist es wichtig und ratsam, den Patienten über diese Gefahr zu informieren und mit ihm dieses Thema rechtzeitig zu besprechen.

Die Frage, ob in einer bestimmten Situation medizinisch etwas getan werden soll oder nicht, muss einem permanenten Such- und Findungsprozess unterworfen sein und erfordert Flexibilität vom Arzt und vom Patienten. Zudem darf ein allfälliger Entscheid nie als grundsätzlich und unumstösslich verstanden werden. Dabei ist zu unterscheiden zwischen (a) dem medizinisch-technischen Tun, für das situativ die Option der Unterlassung besteht, und (b) dem ärztlich-begleitenden Tun. Für Letzteres, namentlich das Präsentsein des Arztes und das Begleiten des Patienten, besteht selbstverständlich nie die Option der Unterlassung. Als Teil der ärztlichen Aufgabe und Pflicht ist ein solches immer geboten. Zudem muss in diesen Such- und Findungsprozess einfliessen, was im ersten Teil dieses Beitrags ausführlich dargestellt wurde. Dazu braucht es die Kenntnis und das Wissen über die drei erwähnten wesentlichen Begrifflichkeiten in der Medizin und deren Bedeutung, und es braucht eine gewisse Sensibilisierung und die Bereitschaft, sich die Auswirkungen und Gefahren dieser Stolpersteine im medizinischen Alltag bewusst zu machen.

Erfahrungen am Krankenbett zeigen, dass der Preis des vermeintlich Machbaren, das sich im Krankheitsverlauf dann doch nicht als machbar erweist, bisweilen wesentlich höher ist, als der Preis, Ungemachtes zu ertragen. Anders gesagt: Die Rechnung geht oft nicht auf, mindestens nicht für den Patienten. 
Es zeigt sich, dass situatives Nicht(s)tun viel zu tun hat mit Ehrlichkeit und Genügsamkeit, beides Tugenden, die im Umgang mit kranken Menschen geboten sind. Zudem ist es Ausdruck von Respekt vor dem Patienten und der Achtung des Prinzips des Nichtschadens, des nihil nocere im Sinne der hippokratischen Tradition. Nachdenken über situatives Nicht(s)tun bedeutet demnach auch Nachdenken über die Frage, wie wir in Zukunft mit den Möglichkeiten der modernen Medizin umzugehen gedenken, und wie sich diese auf den Menschen und die Gesellschaft auswirken wird. Die Entwicklung der Medizin ist nie bloss ein partikulares Geschehen: Neben dem direkten Einfluss auf den Erkrankten hat sie immer auch einen indirekten Einfluss auf die Gesellschaft und letztlich auf die Gattung Mensch.

So stellen sich unter anderem folgende Fragen: Was kann, darf und soll der Mensch mit den exponentiell wachsenden Forschungsergebnissen und den beinahe unbegrenzten Möglichkeiten der Medizin anfangen? Wer hat die Kompetenz, sich hier ein umfassendes Bild zu machen, und wer hat die Weitsicht, das wirklich Sinnvolle und Angemessene in jedem Fall zu erkennen? Je weniger der Mensch bereit ist, das Bestehende und Gewordene zu akzeptieren und vorzugsweise das Machbare sucht, desto mehr wird er zum Zauberlehrling in eigener Sache. «Der Anspruch auf völlige Beherrschung der menschlichen Natur entspringt» - so Klaus Goergen - «einer grandiosen Hybris, die vergessen hat, dass die Seele nicht alles mittragen kann, was der Verstand sich an Freiheit anmasst. Wer sich als Gemachter versteht, wird am Ende als Gemachtes behandelt». ${ }^{13}$

Wir alle - nicht nur wir Ärztinnen und Ärzte - müssen uns mit diesen Themen auseinandersetzen, wir müssen genügend darüber wissen, sachlich argumentieren und letztlich in einer für uns alle verständlichen Sprache Stellung beziehen. Dabei ist zu bedenken, dass unsere Moralvorstellungen sehr verschiedenartig sind. Eine umfassende Argumentation muss sowohl vom konkreten Fall (von

13 Klaus Goergen: Körper und Moral. Medizinische Positionen im Überblick (2008) 1. https: / / docplayer.org/6160045-Koerper-und-moral-medizinethischepositionen-im-ueberblick-2008.html (Zugriff 10.8.2020) 
unten), als auch von allgemeinen ethischen Prinzipien aus (von oben) ein Überlegungsgleichgewicht suchen. Nur so wird es gelingen, intuitive und theoretische Argumente möglichst widerspruchsfrei zusammenzubringen.

2.5 Was tun wir nun aber, wenn wir medizinisch nichts tun?

Angenommen, wir tun medizinisch wirklich nichts. Weder diagnostisch noch therapeutisch, weder betreffend Symptomatik noch Lebensverlängerung. Wenn wir medizinisch nichts tun, nehmen wir keinen Einfluss auf das Profil einer Krankheit und überlassen diese dem Spontanverlauf. Das muss nun aber nicht bedeuten, dass wir überhaupt nichts mehr tun - vielmehr ist etwas ganz anderes zu tun.

Auch wenn es paradox klingen mag: Situatives Nicht(s)tun ist ein aktiver Terminus, denn die Unterlassung medizinischer Interventionen impliziert die Entstehung eines Freiraums, der sinnvoll gefüllt werden kann. Situatives Nicht(s)tun hat gleichsam eine katalytische Wirkung auf eine ganz andere Form und Weise von Tun. Damit kann ein medizinexternes Tun ermöglicht und verwirklicht werden. Ein Handeln also, das sich typischerweise ausserhalb medizinischer Tätigkeiten ansiedelt und mit Medizin gar nichts mehr zu tun hat, umso mehr aber mit einer sinnvollen Beschäftigung mit Dingen und Themen, die sich jenseits des Krankseins verorten. Es ist frei von interventionsassoziierten, unerwünschten Nebenwirkungen und Komplikationen. So geht es letztlich darum, den Übergang von medizininternem zu medizinexternem Tun zu schaffen.

Wenn hier von einer ganz anderen Weise von Tun die Rede ist, so ist damit folgendes gemeint:

- Die Stärkung der Ressourcen des Patienten und die Förderung seiner Resilienz.

- Das Bestreben, die aktuelle gesundheitliche Situation ganz bewusst zu akzeptieren und sich allenfalls durch das Gegebene auch einmal treiben zu lassen.

- Die Beschäftigung mit sich selbst, mit der Familie, dem Freundeskreis - mit Liebhabereien und Vergnügen im Rahmen des Machbaren. 
93 Beat Gerber: Im Spannungsfeld zwischen Tun und Lassen

- Dies alles in Übereinstimmung mit der Persönlichkeit des Betroffenen und mit dessen aktuellen körperlichen und geistigen Möglichkeiten.

Dieser Wechsel von der medizininternen Ebene des Wissenund Handeln-Wollens auf die medizinexterne Ebene der Linderung der individuellen Lebensnot des Patienten lässt erst die Umsetzung des genuin ärztlichen Praktizierens in ihrer Vollständigkeit zu. Der Arzt wird jetzt in einer Weise benötigt, bei der er sich nicht allein durch sein medizinisches Fachwissen und durch medizinischtechnische Anordnungen profilieren kann, sondern in einer Weise, in der er sich dem Patienten als einem Menschen in seinem Leiden stellen muss.

Fazit: Die Thematik des situativen Unterlassens hat in den letzten Jahren zwar Einzug in die medizinischen Publikationen gefunden. Doch sie wird in erster Linie als Sparmassnahme verstanden, als ein Versuch, die Kosten im Gesundheitswesen in den Griff zu bekommen. Dies, ohne dabei zu bedenken, dass die Option der situativen Unterlassung zwingender Bestandteil einer genuinen, patientenorientierten Medizin ist.

Die Legitimität des Tuns, das über Jahrtausende Prämisse und Paradigma zugleich für Erfolg und Prosperität war, sieht sich vermehrt einem ernstzunehmenden Konkurrenten, dem situativen Nicht(s)tun, gegenüber. Sie ist gleichsam das Ergebnis eines philosophischen Denkens, dessen Inhalt und Ziel als Gegenentwurf zu einer Lebensweise zu verstehen ist, die dem Menschen immer mehr zumutet und ihn zunehmend überfordert. Es gilt, in der Medizin die beiden Handlungsoptionen Tun und Nicht(s)tun als gleichwertig zu akzeptieren. Nur so kann eine Entspannung innerhalb des aktuellen Ungleichgewichts in Bezug auf Tun und Lassen in der Medizin gelingen. Verteidigt die Medizin den aktuellen (Hyper-)Aktivismus und gesteht sie diesem weiterhin den prioritären Status zu, besteht die Gefahr, dass der Patient vom Subjekt zum Objekt wird, dass der Kranke gewollt oder ungewollt durch die Medizin instrumentalisiert wird: So beispielsweise, wenn Untersuchungen und Behandlungen durchgeführt werden, die für den Patienten weder diagnostische 
noch therapeutische Konsequenzen haben - Untersuchungen, bei denen bereits prospektiv Nutzen und Sinn in Frage gestellt werden und überdies das Leiden des Patienten noch zusätzlich vergrössern. Oder wenn Therapien installiert werden, die mit schweren Nebenwirkungen verbunden sind, bei denen aber kaum eine Wirkung zu erwarten ist.

Das ärztliche Selbstverständnis erfordert, über althergebrachte, eingefahrene Denkweisen und Mechanismen in der Medizin kritisch nachzudenken. Das Prinzip der heilvollen Unterlassung muss in die erfolgreiche moderne Medizin gewinnbringend integriert werden, aus Respekt vor dem Erkrankten und in Übereinstimmung mit einer zeitgemässen ärztlichen Ethik. Dies im Wissen, dass sich die Medizin nicht immer den moralischen Erwartungen der Gesellschaft unterzieht und dass sie diese Erwartungen auch selbst konstituiert gleichzeitig und nachhaltig.

Es ist deshalb zu hoffen, dass die Gesellschaft dazu bereit ist, dies zu bedenken, und dass es der Politik gelingt, die notwendigen Rahmenbedingungen für das Prinzip der heilvollen Unterlassung zu schaffen. Dazu gehören insbesondere:

(1) ebenbürtige Dignität der beiden Handlungsoptionen

(2) situatives Nicht(s)tun als effektiv disponible Option im Praxisalltag

(3) Befreiung des medizinischen Alltags vor ungesundem (Hyper-)Aktivismus

(4) Bereitschaft, die Risiken und Gefahren zu erkennen, die sich rund um die Thematik von Gesundheit und Krankheit, Medikalisierung und Macht im medizinischen Alltag ergeben

(5) Respektierung des Patienten als Subjekt

(6) Neubeschreibung eines bestmöglichen Arzt-Patienten-Verhältnisses

(7) Bekanntmachung und Ermöglichung einer ganz anderen Art von Tun

Schliesslich darf situatives Nicht(s)tun weder an Bedingungen geknüpft sein, noch darf es Auswirkungen auf Patienten haben, die für diese diskriminierend sind. 
95 Beat Gerber: Im Spannungsfeld zwischen Tun und Lassen

conexus 3 (2020) 75-95

(C) 2020 Beat Gerber. Dieser Beitrag darf im Rahmen der Lizenz CC BY-NCND 4.0 - Creative Commons: Namensnennung/nicht kommerziell/keine Bearbeitungen - weiterverbreitet werden.

(c) $(i)$

https: / / doi.org/10.24445/ conexus.2020.03.007

Dr. Beat Gerber, MAS Philosophie und Medizin, Brechbühlerstrasse 15, 3006 Bern

beat.gerber@hispeed.ch 